\title{
一氧化氮响应性高分子荧光探针的构筑及其细胞内成像应用
}

\author{
郑斌 ${ }^{a}$ 程盛 ${ }^{c}$ 董华泽 ${ }^{a}$ 朱金苗 ${ }^{a}$ 韩钰 $^{a}$ \\ 杨亮 ${ }^{a}$ 胡进明 $*, b$ \\ ( ${ }^{a}$ 合肥师范学院 化学与化学工程学院 合肥 230061) \\ ( ${ }^{b}$ 中国科学技术大学 高分子科学与工程系 合肥 230026) \\ ( ${ }^{c}$ 合肥工业大学 分析测试中心 合肥 230009)
}

\begin{abstract}
摘要 一氧化氮(NO)是一种普遍存在的生理信号分子, 但利用 NO 作为触发方法来精细调节仿生聚合物的自组装行为 的研究却很少. 本工作报道一种独特的具有一氧化氮(NO)反应特性的新型 $\mathrm{pH}$ 响应双亲水性嵌段共聚物(double hydrophilic block copolymer, DHBC), 其中 NO 可以自发地触发聚(寡聚乙二醇甲醚甲基丙烯酸酯)-嵌段-聚(NO 响应性基元共-7-硝基苯并呋咱衍生物)(POEGMA- $b$-P(APUEMA- $c o-\mathrm{NBD})$ )双亲水嵌段共聚物, 分别在酸性和中性环境中发生自组 装和形态转变. 在引入苂光团之后, 这些转变还可以和 $\mathrm{NO}$ 存在下光致诱导电子转移过程被阻断导致荧光增强相关联, 从而提供了观察细胞内 NO 的机会.
\end{abstract}

关键词 一氧化氮; 响应性; 苂光探针; 成像

\section{Construction of Nitric Oxide (NO)-Responsive Fluorescent Polymer and Its Application in Cell Imaging}

\author{
Zheng, $\operatorname{Bin}^{a}$ \\ Cheng, Sheng ${ }^{c}$ \\ Dong, Huaze ${ }^{a}$ \\ Yang, Liang ${ }^{a}$ \\ $\mathrm{Hu}$, Jinming*,b \\ ( ${ }^{a}$ School of Chemistry and Chemical Engineering, Hefei Normal University, Hefei 230061, China) \\ $\left({ }^{b}\right.$ Department of Polymer Science and Engineering, University of Science and Technology of China, Hefei 230026, China) \\ ( ${ }^{c}$ Instrumental Analysis Center, Hefei University of Technology, Hefei 230009, China)
}

\begin{abstract}
Nitric oxide (NO) is a ubiquitous physiological signal messenger, but the use of NO as a trigger event to delicately tune the self-assembly behaviors of biomimetic polymers has been far less exploited. In this work, a single primary amine-containing 2-(3-(2-aminophenyl)ureido)ethyl methacrylate (APUEMA) monomer was first synthesized by the reaction between $o$-phenylenediamine and 2-isocyanatoethyl methacrylate. Then, the well-defined double hydrophilic block copolymer (DHBC), poly[oligo(ethylene glycol)methyl ether methacrylate]-b-poly[2-(3-(2-aminophenyl)ureido)ethyl methacrylate-co-4-(2-methylacryloyloxyethylamino)-7-nitro-2,1,3-benzoxadiazole)] (POEGMA- $b$-P(APUEMA-co-NBD)), was synthesized via sequential reversible addition-fragmentation chain transfer (RAFT) polymerization. Since there is a free amine group in the APUEMA monomer, it can be competent to quench the fluorescence of dyes and react with NO showing NO-responsiveness property. The reaction product of APUEMA and NO was purified by column chromatography, and ${ }^{1} \mathrm{H}$ and ${ }^{13} \mathrm{C}$ NMR results displayed the formation of urea-functionalized benzotriazole residual. The $\mathrm{p} K_{\mathrm{a}}$ values of APUEMA monomer and POEGMA- $b$-P(APUEMA-co-NBD) block polymer were measured to be 3.36 and 2.15 , respectively, indicating that APUEMA monomer and PAPUEMA moieties of POEGMA- $b$-P(APUEMA-co-NBD) showed hydrophilic ability at acidic medium and hydrophobic ability at neutral medium. The aqueous solution of POEGMA- $b$-P(APUEMA-co-NBD) block copolymer exhibited a small diameter with about $5.0 \mathrm{~nm}$ at $\mathrm{pH} 2.0$, which illustrates that block copolymer can dissolve into water with a unimer state. After changing the solution $\mathrm{pH}$ value to 7 , the solution diameter increased to about $10 \mathrm{~nm}$ recorded by dynamic light scattering (DLS). Transmission electron microscope (TEM) results displayed micelles of POEGMA- $b$-P(APUEMA-co-NBD) block copolymer aqueous solution with spherical structures at $\mathrm{pH}$ 7.4. Furthermore, the fluorescence intensity of the block copolymer solution was decreased quickly after the $\mathrm{pH}$ value increased from 2 to 7 . The NO-responsive property of block copolymer POEGMA- $b$-P(APUEMA-co-NBD) was also detected by DLS and fluorescent spectrometry methods. At $\mathrm{pH} 2.0$, the diameter of the block copolymer aqueous solution increased from $5 \mathrm{~nm}$ to about 150 $\mathrm{nm}$ upon sparging with $\mathrm{NO}$ for $24 \mathrm{~h}$. At $\mathrm{pH} 7.0$, the diameter of block copolymer micelles increased from $10 \mathrm{~nm}$ to about 100
\end{abstract}

*E-mail: jmhu@ustc.edu.cn
Received June 30, 2020; published August 21, 2020.

Supporting information for this article is available free of charge via the Internet at http://sioc-journal.cn.

Project supported by the National Natural Science Foundation of China (21504021), the Foundation of Educational Committee of Anhui Province (KJ2019A0719), the Excellent Talent Foundation of Education Department of Anhui Province (gxyq2019066), the Key R\&D Program of Anhui Province (202004a07020020), and the 136 talent plan of Hefei Normal University.

项目受国家自然科学基金(21504021), 安徽省高校自然科学基金重点项目(KJ2019A0719), 安徽省优秀青年项目(gxyq2019066), 安徽省重点研究与开 发项目面上攻关(202004a07020020)和合肥师范学院 136 人才计划项目资助. 
$\mathrm{nm}$ after exposure to NO for $24 \mathrm{~h}$. The transmittance of POEGMA- $b$-P(APUEMA-co-NBD) block copolymer aqueous solution at $\mathrm{pH} 2.0$ or $\mathrm{pH} 7.0$ decreased upon NO addition, which were in accorded with DLS results. Moreover, the fluorescence intensity of the block copolymer solution at $\mathrm{pH} 2.0$ improved rapidly upon sparging with $\mathrm{NO}$ for $0.5 \mathrm{~h}$, implying that the NO-triggered self-assembly of micelles decreased environmental polarity. The fluorescence intensity decreased with further addition. The fluorescence intensity of block copolymer micelles at $\mathrm{pH} 7.0$ exhibited 15 -fold increased after addition with NO for $24 \mathrm{~h}$. The in vitro study of block copolymer POEGMA- $b$-P(APUEMA-co-NBD) was conducted in normal MRC-5 cells. The block copolymer showed negligible cytotoxicity even at the block copolymer concentration of $100 \mathrm{~g} / \mathrm{mL}$. We herein report on a novel $\mathrm{pH}$-responsive $\mathrm{DHBC}$ with unique NO-reactive feature, where NO can spontaneously trigger the self-assembly and morphological transformation in acidic and neutral milieus, respectively. After the introduction of fluorophores, these transitions are also associated with significant fluorescence turn-on due to eliminations of photoinduced electron transfer (PET) process in the presence of NO, imparting the opportunities to visualize intracellular NO.

Keywords nitric oxide; responsive; fluorescent probe; imaging

\section{1 引言}

信号分子在包括免疫、神经和血管系统在内的人类 系统中起着重要的作用 ${ }^{[1-3]}$. 其中一氧化氮(NO)因其与 阿尔茨海默病、癌症和高雪氏病等多种疾病的关系而受 到越来越多的关注 ${ }^{[4-6]}$. NO 作为第一个被发现的信使分 子, 在肌肉松他、免疫系统控制、记忆、血压调节和学 习等方面发挥着重要作用 ${ }^{[7-10]}$. 在活细胞中, $\mathrm{NO}$ 是在诱 导型一氧化氮合酶(inducible nitric oxide synthase, iNOS) 的催化下, 由 $L$-精氨酸转化为 $L$-瓜氨酸的过程中产生 的. 然而, 它极不稳定, 仅在低浓度下存在, 且在不同 浓度范围会显示出不同的药理学活性, 如较低浓度时对 环磷酸鸟苷(cGMP)通路影响而产生舒张血管的作用, 当浓度升高会产生受损机体组织的修复功能, 若继续增 加浓度则会产生细胞调亡行为 ${ }^{[1]]}$. 因此, NO 的检测和 监测对微生物的控制和机体的健康具有重要意义 ${ }^{[12,13]}$. 为了更好地了解和预防相关疾病, 活细胞中 NO 的检测 和监测是非常必要的.

对 NO 的检测已有一些方法. 其中最常用的方案是 基于邻二氨基芳烃和 NO 之间的反应生成三氮唑基团, 这导致苂光团的电子分布变化而产生苂光发射信号的 改变 ${ }^{[14]}$. 利用这一机理, 香豆素在无 $\mathrm{NO}$ 会有光致电子 转移而产生荧光淬灭, 有 $\mathrm{NO}$ 时则产生荧光恢复, 用一 锅法实现了对缺氧和 $\mathrm{NO}$ 双重分析物的检测 ${ }^{[15]}$. 为了提 高穿透能力, 设计了双光子荧光探针, 可以同时吸收两 个近红外光子, 减少部分背景荧光 ${ }^{[16]}$. NO 还可作为金 属中心配合物多元氧化态变化的还原剂, 利用过渡金属 配合物构建了不同的 NO 传感器 ${ }^{[17-21]}$.

研究学者们虽然取得了良好的效果, 但在研究过程 中仍存在一些问题. 如在实验过程中, 酸性条件和细胞 内物质会干扰有机染料的苂光信号, 溶酶体中的水解酶 也限制了其稳定性 ${ }^{[22]}$. 有机小分子探针的溶解性也阻 碍了其在水相中的广泛应用, 且合成难度大, 选择性有 限. 因此, 迫切需要新型的针对 $\mathrm{NO}$ 的检测探针.

Kim 等 ${ }^{[23]}$ 合成了带正电荷的 $N$-(2-氨基苯基)-8-巯基 八酰胺(NAM)修饰的金纳米粒子(AuNPs). NO 的引入诱 导了苯并三坐的形成并导致 8-NAM-AuNP 和带有负电 荷的苯并三唑水解产物的产生. NO 诱导的电荷反转作
用有助于巨噬细胞吞噬组装体, 因此, 进一步的光热治 疗也是可行的. 我们课题组 ${ }^{[24]}$ 开发的聚 $N$-(4-氨基苯基) 甲基丙烯酰胺 ( $p$-NAPMA) 聚合物利用其中含有的苯基 重氮基团具有紫外光敏感性, 在紫外光辐照下可生成邻 苯二胺衍生物, 实现了 $\mathrm{NO}$ 和紫外光(UV)的双重响应. 杨志谋课题组 ${ }^{[25]}$ 以邻苯二氨基为封端基团, 构建了一 个不引入诱导水凝胶转化的四肽超分子水凝胶体系. Nishikawa 等 ${ }^{[26]}$ 设计了一种基于 $N$-烷氧基丙烯酸邻苯二 胺的蛋白质标记的 NO 传感支架.

这些基于高分子聚合物可控设计的理念进行 NO 响 应性聚合物的开发与利用是比较有成效的, 然而鉴于 $\mathrm{NO}$ 在体内的重要性, 对可以进行体内原位检测和监测 的 NO 响应性聚合物的构筑是很有必要的. 通过侧链修 饰及共聚合的方式将具有 $\mathrm{NO}$ 响应性的基元修饰在大分 子上, 利用大分子在溶液中的组装特性产生具有响应性 的胶束结构. 这种胶束组装的纳米颗粒可在水溶液中分 散实现水溶液体系的 $\mathrm{NO}$ 监测同时纳米颗粒的产生又可 有效避免外排作用的发生提高检测效率.

在本工作中我们采用可逆加成断裂链转移自由基 聚合方法(RAFT)合成了具有良好 $\mathrm{pH}$ 和 NO 双响应性的 双亲水性嵌段共聚物 POEGMA- $b$-P(APUEMA- $c o-$ NBD), 其中 OEGMA、APUEMA 和 NBD 分别为寡聚乙 二醇甲醚甲基丙烯酸酯、基于邻苯二胺的 $\mathrm{NO}$ 反应性单 体和 7-硝基苯呋咱衍生物. 由于聚合物中含有的 APUEMA 单体单元存在一个自由胺基，其能够通过光 诱导电子转移(PET)过程使染料荧光淬灭, 而在 NO 存 在情况下消除了此过程, 因此可以产生苂光打开的响应 效果, 响应示意图和聚合物合成路线如图式 1 和图式 2 所示.

\section{2 结果与讨论}

\section{1 单体响应性与聚合物结构表征}

通过邻苯二胺与甲基丙烯酸异氧基乙酯的反应, 合 成了一种含伯胺的 APUEMA 单体. ${ }^{1} \mathrm{H}$ 和 ${ }^{13} \mathrm{C} N \mathrm{NR}$ 分析 表明 APUEMA 单体被成功地制备出来(图 1a, 1b). 也获 得了不含邻苯二胺基的 2-(3-苯基脲基)甲基丙烯酸乙酯 (PUEMA) 作为对照 (图 S1). 为了研究 APUEMA 和 


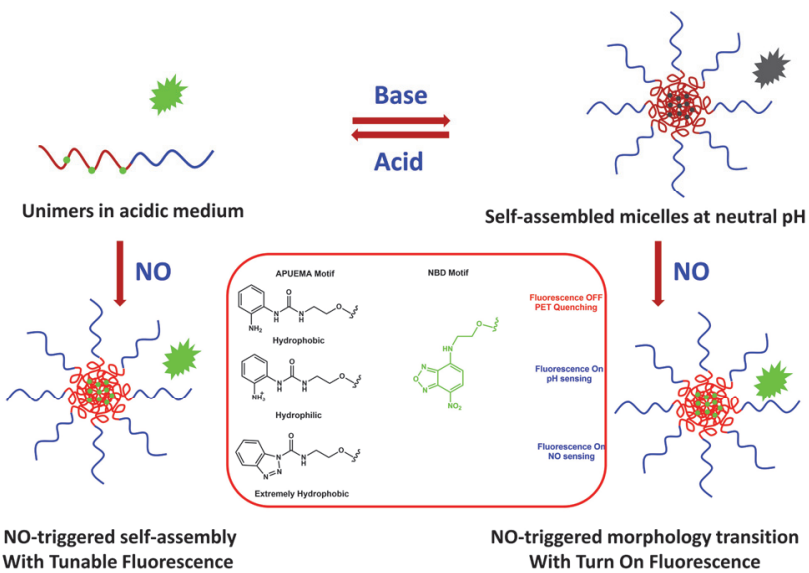

图式 1 POEGMA- $b-\mathrm{P}(\mathrm{APUEMA}-c o-\mathrm{NBD})$ 在 NO 作用下响应示意图. Scheme 1 Illustration of POEGMA- $b$-P(APUEMA-co-NBD) responsiveness to NO.

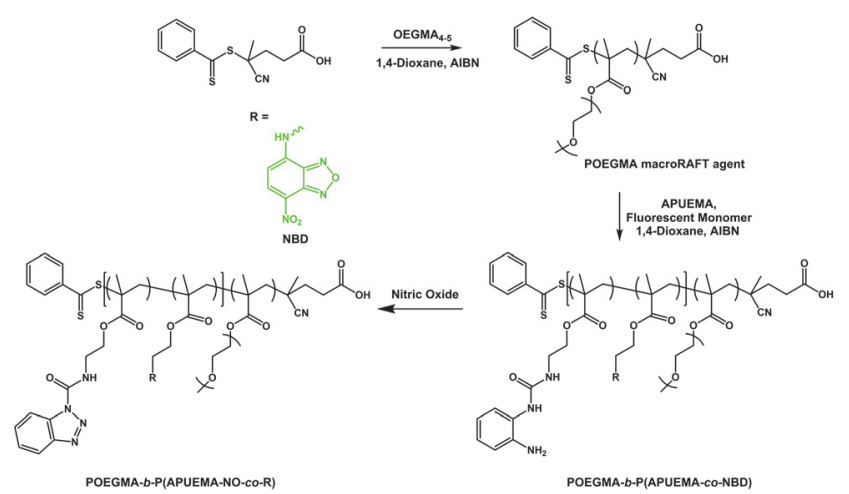

图式 2 POEGMA- $b$-P(APUEMA-co-NBD)合成路线图.

Scheme 2 Synthetic route of POEGMA- $b$-P(APUEMA-co-NBD).

PUEMA 对 NO 的响应性, 在不同 NO 浓度下, 对单体水 溶液进行了 UV/Vis 光谱监测, 发现 APUEMA 水溶液在 $258 \mathrm{~nm}$ 和 $295 \mathrm{~nm}$ 处出现两个吸收峰, 并随着 $\mathrm{NO}$ 浓度 的提高紫外吸收峰的强度也逐渐增强(图 4a, 4b). 然而, 加入 NO 后, PUEMA 水溶液的吸收强度没有明显变化, 表明 PUEMA 对 NO 没有响应性(图 4c). 据报道邻苯二 胺基团在温和条件下可以与 $\mathrm{NO}$ 反应生成苯并三唑, 并 具有较高的转化率. 以柱层析法纯化 APUEMA 和 NO 的反应产物, ${ }^{1} \mathrm{H}$ 和 ${ }^{13} \mathrm{C} \mathrm{NMR}$ 结果显示产物具有脲基官能 化的苯并三唑残留(图 2a，2b). 然后，通过 RAFT 聚合， 合成了一种性能良好的双亲水嵌段共聚物 POEGMA- $b$-P(APUEMA-co-NBD). 根据 ${ }^{1} \mathrm{H}$ NMR 谱图, POEGMA 和 PAPUEMA 的聚合度(DPs)均为 13(图 3). 从 $\mathrm{GPC}$ 的结果可以看到 POEGMA- $b$-P(APUEMA-co$\mathrm{NBD})$ 嵌段共聚物具有单分散性, 分子量 $\left(M_{\mathrm{n}}\right)$ 为 11.7 $\mathrm{kDa}$, 多分散性 $\left(M_{\mathrm{w}} / M_{\mathrm{n}}\right)$ 为 1.28 (图 S2). GPC 结果表明, POEGMA- $b$-P(PUEMA- $c o-\mathrm{NBD})$ 嵌段共聚物可以通过 可控聚合获得, 其 $M_{\mathrm{n}}$ 和 $M_{\mathrm{w}} / M_{\mathrm{n}}$ 分别为 $22.8 \mathrm{kDa}$ 和 1.10(图 S3).
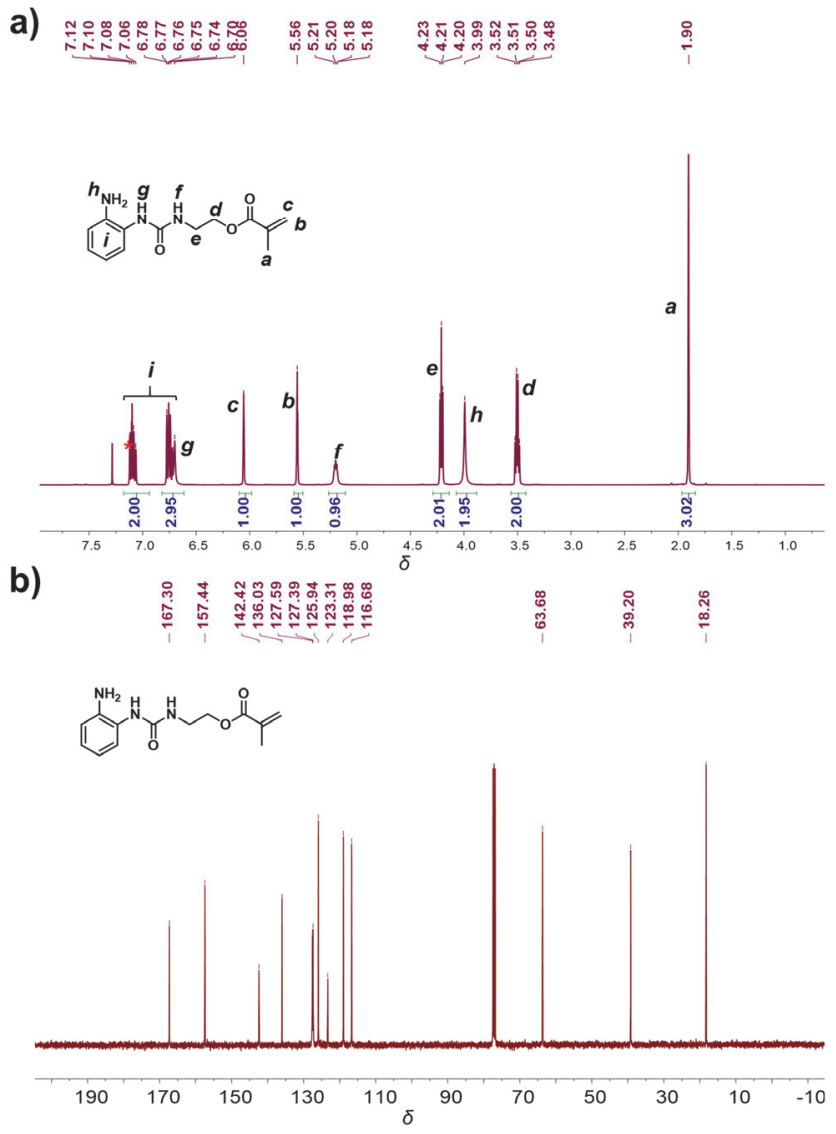

图 1 APUEMA 的(a) ${ }^{1} \mathrm{H}$ 和(b) ${ }^{13} \mathrm{C}$ 核磁共振谱图, 氛代氯仿作为溶剂. Figure 1 (a) ${ }^{1} \mathrm{H}$ NMR and (b) ${ }^{13} \mathrm{C}$ NMR spectra of APUEMA in $\mathrm{CDCl}_{3}$.

\section{2 聚合物的 $\mathrm{pH}$ 性能表征}

采用电位滴定法、动态光散射法和苂光光谱法研究 了嵌段共聚物 POEGMA- $b$-P(APUEMA- $c o$-NBD)的 $\mathrm{pH}$ 响应特性。测定了 APUEMA 单体和 POEGMA- $b$ P(APUEMA-co-NBD) 嵌段聚合物的 $\mathrm{p} K_{\mathrm{a}}$ 值分别为 3.36 和 2.15, 表明 POEGMA- $b$-P(APUEMA-co-NBD) 的 PAPUEMA 部分在酸性介质中具有亲水性, 在中性介质 中具有疏水性 (图 S4). POEGMA- $b$-P(APUEMA-coNBD) 嵌段共聚物的水溶液在 $\mathrm{pH} 2.0$ 时直径较小, 约为 $5.0 \mathrm{~nm}$, 说明嵌段共聚物能以单链状态溶解于水中. 将 溶液 $\mathrm{pH}$ 值改为 7 后, 动态光散射记录的流体力学直径 增加到约 $10 \mathrm{~nm}$ (图 5a). TEM 结果显示, 在 $\mathrm{pH} 7.4$ 时, POEGMA- $b$-P(APUEMA- $c o-\mathrm{NBD})$ 嵌段共聚物水溶液的 胶束呈球形结构(图 5b). 胶束的形成是由于 PAPUEMA 部分的去质子使其由亲水性向疏水性的转变引起的自 组装. 此外, 当 $\mathrm{pH}$ 值从 2 增加到 7 时, 嵌段共聚物溶液 的荧光强度迅速降低, 这归因于通过光诱导电子转移 (PET)机制，去质子的 PAPUEMA 部分作为 PNBD 的苂 光猝灭剂而产生苂光淬灭效应(图 5c,5d). 
a)
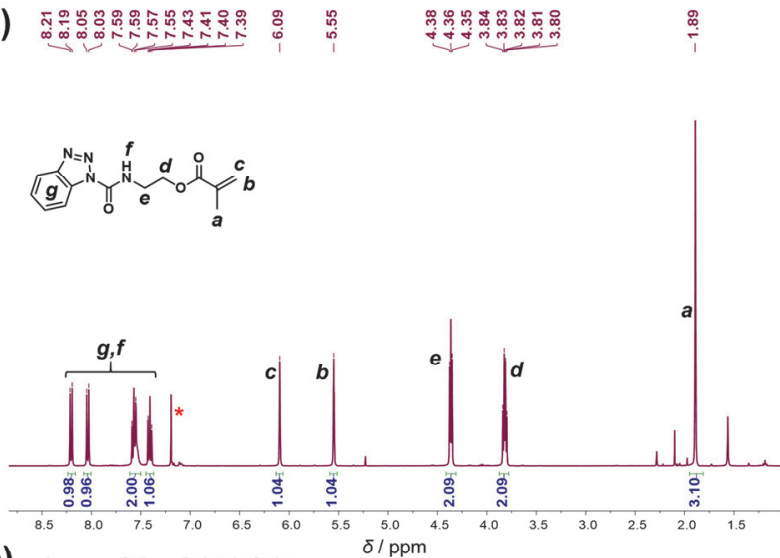

b)
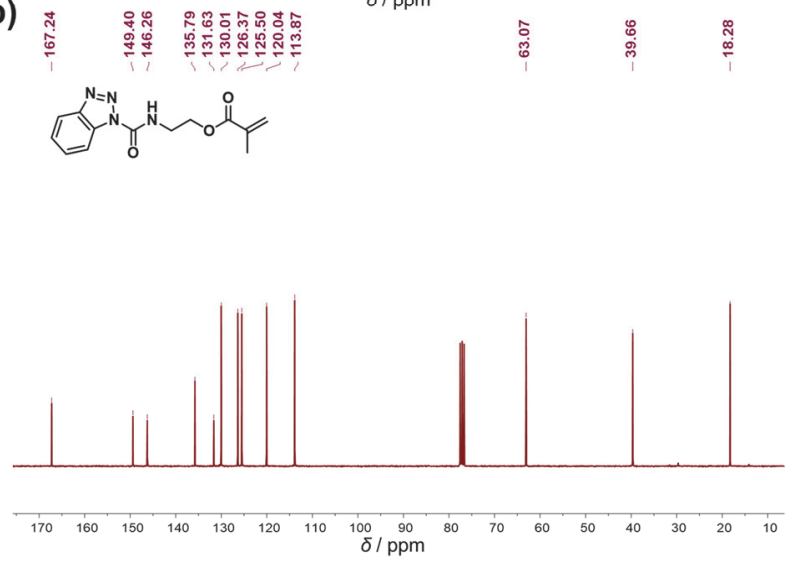

图 2 APUEMA 与 $\mathrm{NO}$ 反应后产物的(a) ${ }^{1} \mathrm{H}$ (氞代 DMSO)和(b) ${ }^{13} \mathrm{C}($ 氞 代氯仿)核磁共振谱图.

Figure 2 (a) ${ }^{1} \mathrm{H}$ NMR (in DMSO- $d_{6}$ ) and (b) ${ }^{13} \mathrm{C}$ NMR (in $\mathrm{CDCl}_{3}$ ) spectra after APUEMA reaction with NO.

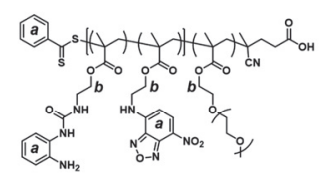

b)

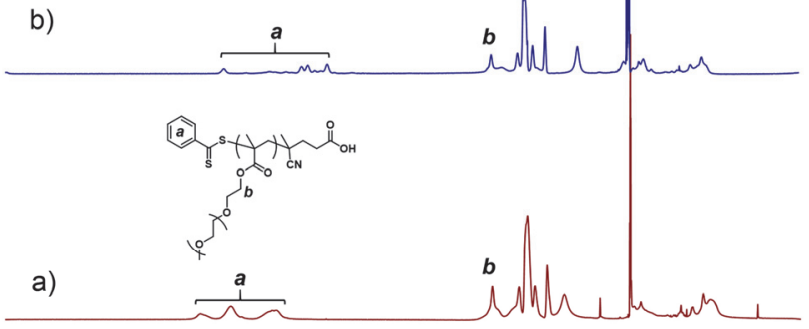

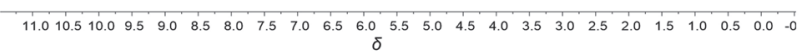

图 3 (a) POEGMA 大分子链转移剂和 (b) POEGMA- $b$ P(APUEMA-co-NBD)两嵌段共聚物在氛代丙酮的 ${ }^{1} \mathrm{H}$ 核磁共振谱图.

Figure $3{ }^{1} \mathrm{H}$ NMR spectra recorded in acetone- $d_{6}$ for (a) POEGMA macroRAFT agent and (b) POEGMA- $b$-P(APUEMA-co-NBD) diblock copolymer.

\section{3 聚合物的 NO 响应性能表征}

用 D L S 和苂光光谱法检测了嵌段共聚物
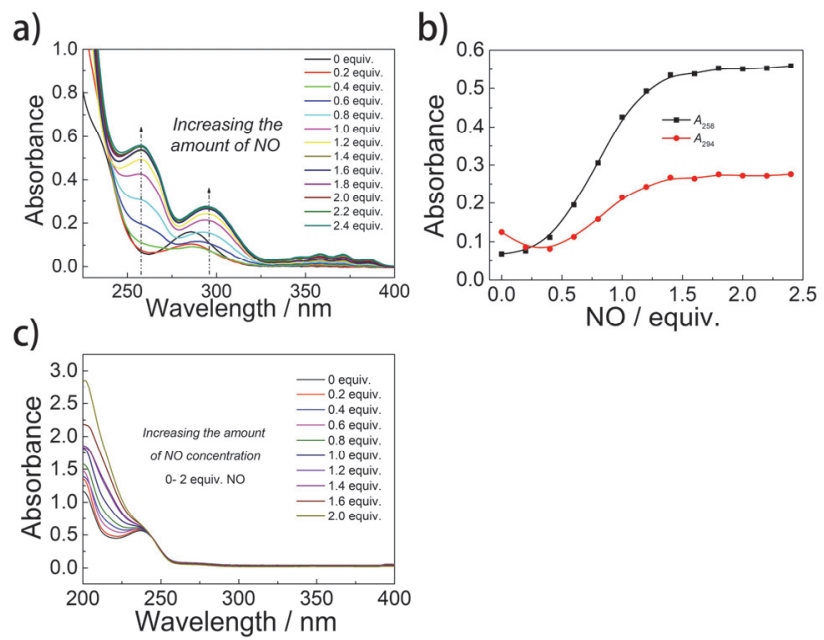

图 4 APUEMA (a, b)和 PUEMA (c)单体溶液在不同浓度 NO 下的紫 外/可见吸收光谱 $(\mathrm{a}, \mathrm{c})$ 和吸收强度变化图(b).

Figure 4 (a, c) UV/Vis spectra and (b) absorption intensity changes recorded for aqueous solutions of the APUEMA (a, b) and PUEMA (c) monomer solution upon addition with various concentrations of $\mathrm{NO}$. a)

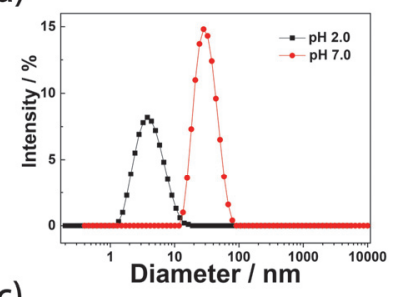

c)

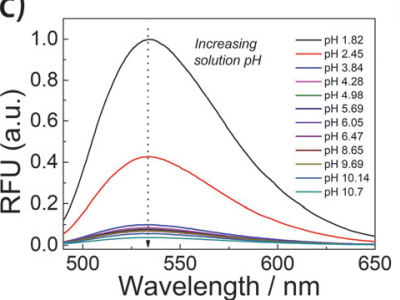

b)

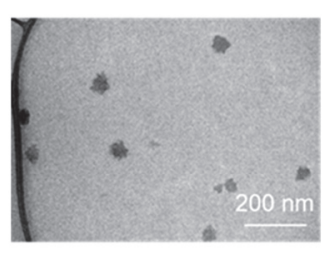

d)

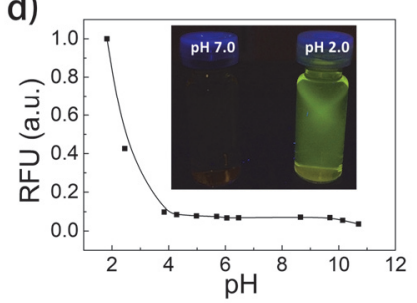

图 5 (a) POEGMA- $b$-P(APUEMA- $c o-\mathrm{NBD}$ )水溶液在不同 $\mathrm{pH}$ 下的动 态光散射图, (b) POEGMA- $b$-P(APUEMA-co-NBD)水溶液在 pH7.4 条 件下的透射电子显微镜图, (c) POEGMA- $b$-P(APUEMA- $c o-N B D)$ 水溶 液 $(1.0 \mathrm{~g} / \mathrm{L})$ 随 $\mathrm{pH}$ 变化的苂光光谱图, (d) POEGMA- $b$-P(APUEMA$c o-\mathrm{NBD})$ 水溶液的归一化荧光强度随 $\mathrm{pH}$ 的变化曲线, 插图为 POEGMA- $b$-P(APUEMA- $c o$-NBD) 水溶液在 $\mathrm{pH} 2.0$ 和 $\mathrm{pH} 7.0$ 条件下的 宏观照片.

Figure 5 (a) Typical DLS profile of POEGMA- $b$-P(APUEMA$c o-\mathrm{NBD}$ ) aqueous solution at different $\mathrm{pH}$ values, (b) transition electron microscopy (TEM) image of the POEGMA- $b$-P(APUEMA-co-NBD) aqueous solution at $\mathrm{pH} 7.4$, (c) normalized $\mathrm{pH}$-dependent fluorescence spectra and (d) fluorescence intensity changes of POEGMA- $b$ $\mathrm{P}(\mathrm{APUEMA}-\mathrm{co}-\mathrm{NBD})$ aqueous solution. The inset macroscopic image in (d) shows the aqueous solution of POEGMA- $b$-P(APUEMA-co-NBD) at $\mathrm{pH} 2.0$ or $\mathrm{pH} 7.0$.

POEGMA- $b$-P(APUEMA-co-NBD)的 NO 响应性. 在 $\mathrm{pH}$ 2.0 时, NO 充入 $24 \mathrm{~h}$ 后, 嵌段共聚物水溶液的直径从 5 $\mathrm{nm}$ 增加到约 $150 \mathrm{~nm}$ (图 6a). PAPUEMA 与 NO 反应后生 成的疏水性嫝基官能化苯并三唑部分，可能导致两亲性 嵌段共聚物的自组装和胶束的膨胀，从而产生大尺寸的 
聚集体. 在 $\mathrm{pH} 7.0$ 时, 暴露于 NO $24 \mathrm{~h}$ 后, 嵌段共聚物 胶束的直径从 $10 \mathrm{~nm}$ 增加到约 $100 \mathrm{~nm}$ (图 6b). 结果表明, 与 PAPUEMA 相比, 䐖官能化的苯并三唑部分具有更高 的疏水性, 加入 NO 后胶束的溶胀度增大. POEGMA- $b-$ P(APUEMA-co-NBD) 嵌段共聚物水溶液在 $\mathrm{pH} 2.0$ 或 $\mathrm{pH}$ 7.0 时的透光率随着 NO 的加入降低符合 DLS 结果(图 S5). 此外, 在 $\mathrm{pH}$ 值为 2.0 的嵌段共聚物溶液中, 加入 NO 后 $0.5 \mathrm{~h}$ 苂光强度迅速提高, 说明 NO 引发的胶束自 组装降低了环境极性. 然后, 随着进一步 $\mathrm{NO}$ 的添加, 苂光强度降低(图 7a, 7b). 在 $\mathrm{pH} 7.0$ 条件下, 加入 $\mathrm{NO}$ 后, 嵌段共聚物胶束的苂光强度增加了 15 倍, 这主要归因 于 PAPUEMA 和 PNBD 之间的 PET 效应减弱(图 7c, 7d). 结果表明, $\mathrm{pH}$ 值的变化或 $\mathrm{NO}$ 的加入均可引发嵌段共聚 物 POEGMA- $b$-P(APUEMA-co-NBD)的自组装和形态转 变. a)

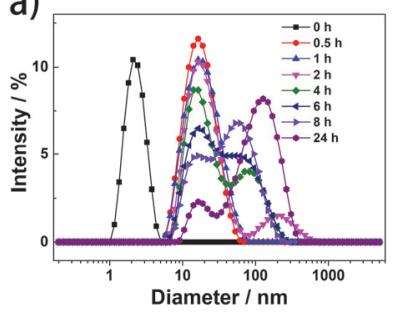

b)

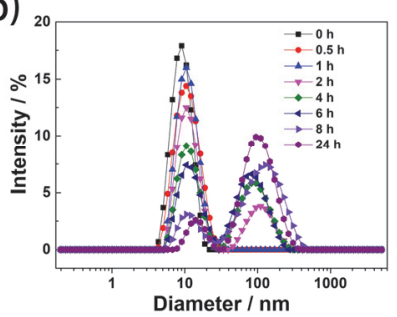

图 6 POEGMA- $b-\mathrm{P}(\mathrm{APUEMA}-\mathrm{co}-\mathrm{NBD})$ 水溶液与 $\mathrm{NO}$ 反应后在(a) $\mathrm{pH}$ 2.0 条件下和(b) $\mathrm{pH} 7.0$ 条件下尺寸随时间的变化关系

Figure 6 Time-dependent size changes of POEGMA- $b$-P(APUEMA$c o-\mathrm{NBD}$ ) aqueous solution at (a) $\mathrm{pH} 2.0$ and (b) $\mathrm{pH} 7.0$ after reaction with NO. a)

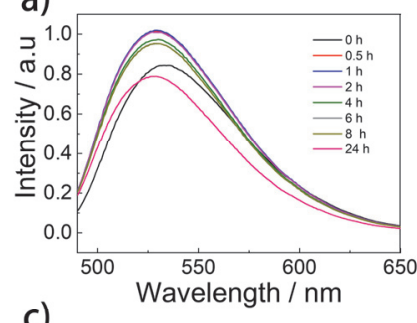

C)

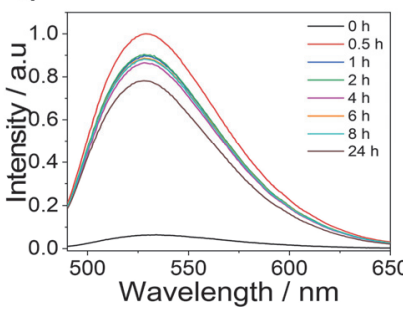

b)

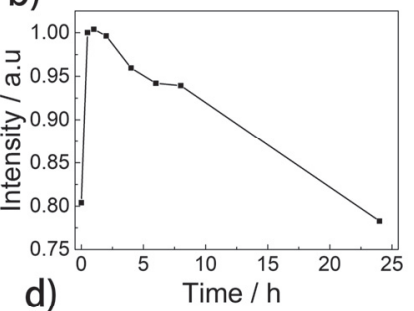

d)

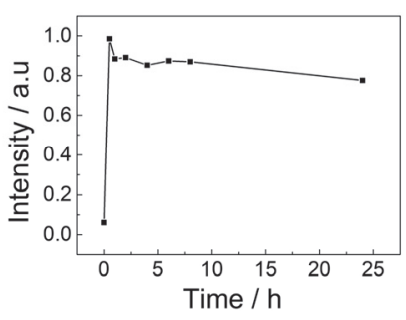

图 7 POEGMA- $b-\mathrm{P}($ APUEMA- $c o-\mathrm{NBD})$ 水溶液与 $\mathrm{NO}$ 反应后在 $\mathrm{pH} 2.0$ $(a, b)$ 和 pH $7.0(c, d)$ 条件下随时间的荧光光谱图 (a, c) 和随时间的苂光 强度变化图(b, d)

Figure 7 Normalized time-dependent fluorescence spectra $(a, c)$ and fluorescence intensity $(\mathrm{b}, \mathrm{d})$ of POEGMA- $b$-P(APUEMA-co-NBD) aqueous solution at $\mathrm{pH} 2.0(\mathrm{a}, \mathrm{b})$ and $\mathrm{pH} 7.0(\mathrm{c}, \mathrm{d})$ after reaction with $\mathrm{NO}$.

\section{4 聚合物的细胞内表征}

在正常 MRC-5 细胞中进行了嵌段共聚物 POEGMA- $b$-P(APUEMA- $c o-N B D)$ 和 POEGMA- $b$ P(PUEMA-co-NBD)的体外研究. 即使在 $100 \mu \mathrm{g} / \mathrm{mL}$ 的 嵌段共聚物浓度下，两种嵌段共聚物也只是显示出可忽 略的细胞毒性(图 S6). 由共聚焦显微镜图片可以看到, 单纯的聚合物 POEGMA- $b$-P(APUEMA- $c o-N B D)$ 在细胞 中无明显苂光，当 $\mathrm{NO}$ 存在时会有很明显的苂光出现， 展现 turn-on 检测效果且对 NO 具有实时检测性. 在对比 聚合物 POEGMA- $b$-P(PUEMA-co-NBD)中即使没有 NO 也有强烈的荧光，说明了没有邻苯二氨基的自由氨基的 存在, PET 效应无法实现, 因此苂光正常显现, 如图 8 所 示. a)

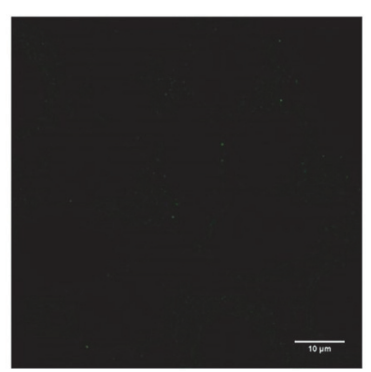

C)

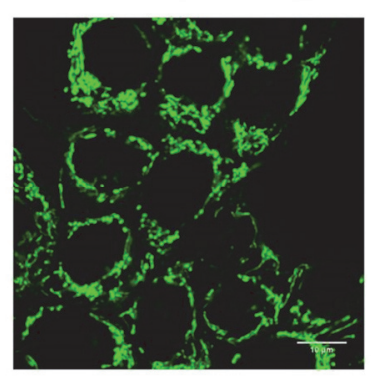

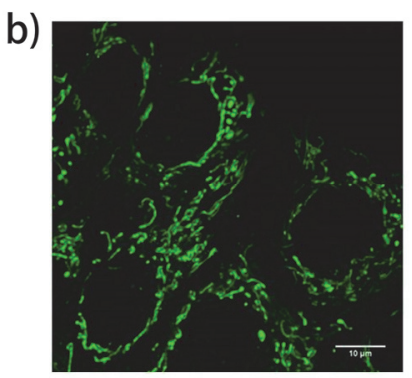

图 8 MCF-7 细胞与纳米粒子孵育后的共聚焦显微镜图. (a) POEGMA$b$-P(APUEMA-co-NBD) 纳米粒子, (b) NO 预处理和 POEGMA- $b$ P(APUEMA-co-NBD)纳米粒子, (c) POEGMA- $b$-P(PUEMA-co-NBD) 纳米粒子.

Figure 8 Confocal laser scanning microscopy images of MCF-7 cells after incubation with nanoparticles. (a) POEGMA- $b$-P(APUEMA$c o$-NBD) nanoparticles, (b) POEGMA- $b$-P(APUEMA-co-NBD) nanoparticles with NO pretreatment, (c) POEGMA- $b$-P(PUEMA-co-NBD) nanoparticles.

\section{3 结论}

综上所述，我们合成了具有 $\mathrm{pH}$ 和 NO 响应特性的 双亲水嵌段共聚物 POEGMA- $b$-P(APUEMA- $c o-N B D)$. 嵌段共聚物 POEGMA- $b$-P(APUEMA- $c o-N B D)$ 溶液的自 组装和形态转变可以通过提高 $\mathrm{pH}$ 值或加入 $\mathrm{NO}$ 来实现, 由于 $\mathrm{NO}$ 的加入可以调节组装体的苂光强度, 因此嵌段 共聚物 POEGMA- $b$-P(APUEMA- $c o-\mathrm{NBD})$ 可以作为检测 细胞内 NO 的传感器进行使用. 


\section{4 实验部分}

\section{1 试剂与仪器}

邻苯二胺、盐酸 $(2 \mathrm{~mol} / \mathrm{L} \mathrm{HCl}$ 的乙醚溶液)、甲基丙 烯酸异氰基乙酯、4-氰基-4-(硫代苯甲酰)戊酸(CPPA)和 2,2'-偶氮二异丁腈(AIBN)均购于 Aldrich. 聚乙二醇甲醚 甲基丙烯酸酯(数均聚合度 300)在使用前先用阻聚剂移 除柱纯化并在 $-20{ }^{\circ} \mathrm{C}$ 下储存. 亚硝酸钠 $\left(\mathrm{NaNO}_{2}\right)$ 和盐 酸溶液 $(37 \%)$ 均购买于 Ajax Finechem 并直接使用. 根据 文献报道的方法合成了 4-(2-甲基丙烯酰氧乙基氨基)-7硝基-2,1,3-苯并噁二唑(NBD). 将 $2.0 \mathrm{~mol} / \mathrm{L} \mathrm{HCl}$ 水溶液 缓慢滴入含有饱和 $\mathrm{NaNO}_{2}$ 溶液的玻璃瓶中, 可产生 $\mathrm{NO}$. 制备饱和 $\mathrm{NO}$ 溶液 $\left(1.8 \mathrm{mmol} / \mathrm{L}, 20{ }^{\circ} \mathrm{C}\right)$, 并使用文献的 方法测定最终浓度.

\section{2 实验合成过程}

制备 POEGMA 大分子链转移剂和 POEGMA- $b$ P(APUEMA- $c o-N B D)$ 双亲水嵌段共聚物所采用的合成 方案如图式 2 所示.

\subsubsection{APUEMA 单体的合成}

根据我们报道的方法合成了去质子化的 APUEMA 单体. 邻苯二胺 $(2.7 \mathrm{~g}, 25 \mathrm{mmol})$ 溶解于 $20 \mathrm{~mL}$ 无水乙腈 中. 将甲基丙烯酸异氰基乙酯(3.88 $\mathrm{g}, 25 \mathrm{mmol}$ )溶解于 $20 \mathrm{~mL}$ 无水乙腈中并逐滴添加. 将混合物在室温下进一 步搅拌过夜. 之后, 在真空下去除乙腈, 残余物用过量 的乙醚沉淀. 采用柱层析法分离纯化不溶性固体. 得到 的去质子化 APUEMA 单体为白色固体(4.9 g, 18.6 mmol). ${ }^{1} \mathrm{H}$ NMR $\left(400 \mathrm{MHz}, \mathrm{CDCl}_{3}\right) \delta: 7.20 \sim 6.98(\mathrm{~m}$, $2 \mathrm{H}), 6.83 \sim 6.68(\mathrm{~m}, 3 \mathrm{H}), 6.06(\mathrm{~s}, 1 \mathrm{H}), 5.56(\mathrm{~s}, 1 \mathrm{H}), 5.20$ (t, 1H, $J=6.0 \mathrm{~Hz}), 4.21(\mathrm{t}, J=5.5 \mathrm{~Hz}, 2 \mathrm{H}), 3.99(\mathrm{~s}, 2 \mathrm{H})$, 3.50 (q, $J=5.7 \mathrm{~Hz}, 2 \mathrm{H}), 1.90$ (s, 3H). ${ }^{13} \mathrm{C} \mathrm{NMR}\left(\mathrm{CDCl}_{3}\right) \delta$ : $167.30,157.44,142.42,136.02,127.59,127.39,125.94$, $123.31,118.98,116.68,63.68,39.20,18.26$.

\subsection{2 中性 $\mathrm{pH}$ 下用 NO 处理 APUEMA 单体}

APUEMA 单体 $(78 \mathrm{mg}, 0.3 \mathrm{mmol})$ 溶解于去离子水/ 乙腈 $(20 \mathrm{~mL}, V / V=1: 1)$ 中. 通入 $\mathrm{NO}$ 气体 $1 \mathrm{~h}$, 然后在 真空下去除乙腈. 将水溶液冷冻干燥, 以二氯甲烷为洗 脱剂, 采用柱层析法纯化最终产物, 产物为白色固体 (78 mg, 收率约 85\%). ${ }^{1} \mathrm{H}$ NMR (400 $\left.\mathrm{MHz}, \mathrm{CDCl}_{3}\right) \delta$ : $8.20(\mathrm{~d}, J=8.3 \mathrm{~Hz}, 1 \mathrm{H}), 8.04(\mathrm{~d}, J=8.4 \mathrm{~Hz}, 1 \mathrm{H}), 7.64 \sim$ $7.51(\mathrm{~m}, 1 \mathrm{H}), 7.41(\mathrm{dd}, J=8.4,6.0 \mathrm{~Hz}, 1 \mathrm{H}), 6.09$ (s, 1H), $5.55(\mathrm{~s}, 1 \mathrm{H}), 4.36(\mathrm{dd}, J=5.8,4.9 \mathrm{~Hz}, 3 \mathrm{H}), 3.82(\mathrm{dd}, J=$ 4.8, $5.9 \mathrm{~Hz}, 2 \mathrm{H}), 1.89$ (s, 2H). ${ }^{13} \mathrm{C} \mathrm{NMR}\left(\mathrm{CDCl}_{3}\right)$ : 167.22, $149.38,146.26,135.78,131.62,130.03,126.39,125.50$, $120.05,113.87,63.07,39.65,18.29$.

\subsubsection{RAFT 聚合法合成 POEGMA 大分子链转移剂}

将 OEGMA (6.0 g, $20 \mathrm{mmol}) 、$ CPPA (280 mg, 1 $\mathrm{mmol}) 、 \operatorname{AIBN}(16.4 \mathrm{mg}, 0.1 \mathrm{mmol})$ 和甲苯 $(12 \mathrm{~mL})$ 装入聚
合小瓶用磁子搅拌. 将混合物脱气 $30 \mathrm{~min}$, 然后置于 $70{ }^{\circ} \mathrm{C}$ 的油浴中开始聚合. $6 \mathrm{~h}$ 后, 聚合反应在冰水浴中 淬灭并用过量的乙醚沉淀. 二氯甲烷中溶解后，进行溶 解-沉淀循环重复三次. POEGMA 大分子 RAFT 试剂在 离心 $5000 \mathrm{r} / \mathrm{min}, 5 \mathrm{~min}$ 的条件下分离, 并在真空烘箱中 在室温下干燥过夜. 获得红色粘稠液体, 通过核磁共振 氢谱测定聚合度为 13 .

4.2.4 用 POEGMA 大分子链转移剂合成 POEGMA- $b$ P(APUEMA-co-NBD) 双亲水性嵌段共聚物

采用 RAFT 法合成 POEGMA- $b$-P(APUEMA-coNBD) 双亲水性嵌段共聚物. 将 POEGMA 大分子 RAFT 试剂(0.41 g, $0.1 \mathrm{mmol}) 、 A P U E M A(0.526 \mathrm{~g}, 2 \mathrm{mmol}) 、$ NBD (5.8 mg, $0.02 \mathrm{mmol}) 、 A I B N(2 \mathrm{mg}, 12 \mu \mathrm{mol})$ 和 DMF $(4.0 \mathrm{~mL})$ 装入带有磁子的聚合瓶中. 将混合物脱气 30 $\min$, 然后置于 $70{ }^{\circ} \mathrm{C}$ 的油浴中开始聚合. $16 \mathrm{~h}$ 后, 聚合 反应在冰水浴中淬灭, 并通过丙酮透析纯化. 新鲜丙酮 每 $4 \mathrm{~h}$ 更换一次. $24 \mathrm{~h}$ 后, 利用过量的乙醚再对嵌段共聚 物进行沉淀. 得到的 POEGMA- $b$-P(APUEMA-co-NBD) 二嵌段共聚物为黄色固体.

\subsection{POEGMA- $b-P(A P U E M A-c o-N B D)$ 双亲水性嵌段 共聚物的自组装}

以二嵌段共聚物为原料, 通过 $\mathrm{pH}$ 调节或共溶剂法 制备了自组装胶束. 溶液 $\mathrm{pH}$ 调整法: 聚合物 $(10 \mathrm{mg})$ 溶 于 $10 \mathrm{~mL}$ 去离子水中, $\mathrm{pH}$ 值调整为 2.0. 用去离子水 $(\mathrm{pH}$ 6.8) 对酸性溶液进行彻底透析, 直至溶液 $\mathrm{pH}$ 值约为 6.5. 将所得溶液稀释至所需浓度以供进一步研究.

共溶剂法: 将聚合物(5 mg)溶解在 $1 \mathrm{~mL} \mathrm{DMF}$ 和去 离子水 $(1 \mathrm{~mL})$ 中, 并在 $1 \mathrm{~h}$ 内通过注射器泵缓慢添加去 离子水 $(1.0 \mathrm{~mL})$. 将混合物进一步搅拌 $1 \mathrm{~h}$, 并加入 8.0 $\mathrm{mL}$ 去离子水. 将聚合物溶液用去离子水透析 $24 \mathrm{~h}$ (MWCO $3.5 \mathrm{kDa})$, 每隔 $6 \mathrm{~h}$ 更换一次新鲜去离子水. 最 后, 将胶体分散液稀释至所需浓度以供进一步实验.

\section{4 聚合物表征}

\subsection{1 核磁共振(NMR)谱}

所有的核磁共振氢谱和碳谱都从 Bruker-AC300F(300 MHz) 谱仪上记录. 以㲴代氯仿 $\left(\mathrm{CDCl}_{3}\right)$ 、気代 DMSO 和気代丙酮为溶剂.

\subsection{2 体积排除色谱法(SEC)}

使用溴化锂 $(0.03 \%, w / V)$ 及 2,6 -二丁基-4-甲基苯酚 (BHT) $(0.05 \%, w / V)$ 处理过的 $N, N$-二甲基乙酰胺(DMAC) 作为溶剂进行测试. 使用岛津模块化系统对聚合物样品 进行 SEC 分析, 该系统包括 DGU-12A 脱气器、 SIL-10AD 自动注射器、 $5.0 \mu \mathrm{m}$ 粒径保护柱 $(50 \mathrm{~mm} \times 7.8$ $\mathrm{mm}$ )、4 个 $300 \mathrm{~mm} \times 7.8 \mathrm{~mm}$ 线性酚醛树脂凝胶柱(微球 尺寸: $5.0 \mu \mathrm{m}$; 孔径: $105 、 104 、 103$ 和 $500 \AA ̊$ ) 和 RID-10A 差分折射率检测器. 使用 CTO-10A 烘箱将色谱柱温度 保持在 $50{ }^{\circ} \mathrm{C}$, 使用 LC-10AT 泵将流速保持在 $1 \mathrm{~mL} / \mathrm{min}$. 
使用分子量范围为 500 至 $10^{6} \mathrm{~g} / \mathrm{mol}$ 的商用窄分子量分 布聚苯乙烯标准品绘制分子量校准曲线. 在洗脱液中制 备 $2 \sim 3 \mathrm{mg} / \mathrm{mL}$ 的聚合物溶液, 并在注入前通过 $0.45 \mu \mathrm{m}$ 过滤膜过滤.

\subsection{3 动态光散射(DLS)}

DLS 的测量是使用运行 DTS 软件的 Malvern Zetasizer 纳米系列, 使用波长为 $633 \mathrm{~nm}$ 的 $4 \mathrm{~mW} \mathrm{He-Ne}$ 激光器和雪崩光电二极管(APD)探测器进行的. 散射光 检测角度为 $173^{\circ}$. 温度稳定在设定温度的 $\pm 0.1{ }^{\circ} \mathrm{C}$. 在 测量之前, 通过 $0.45 \mu \mathrm{m}$ 孔径过滤膜过滤聚合物样品的 水溶液以去除灰尘. 为了评估尺寸分布, 用累积量法拟 合自相关函数.

\subsection{4 透射电子显微镜(TEM)}

在 $200 \mathrm{kV}$ 加速电压下, 用透射电子显微镜观察了 星形聚合物的尺寸和形貌. 聚合物聚集体沉积在 300 目 多孔膜铜栅(Pro Sci Tech)上.

\subsection{5 荧光测量}

苂光发射光谱在 Perkin-Elmer 苂光光谱仪上以 “En Spire” 模式固定于 $470 \mathrm{~nm}$ 激发波长进行测定.

\subsection{6 紫外可见吸收测量}

紫外-可见光谱在 Varian Cary 300 紫外-可见分光光 度计上进行测试, 使用光程为 $10 \mathrm{~mm}$ 的石英比色血作为 容器.

\subsection{7 浊度测量}

采用岛津紫外 2600 分光光度计, 在波长为 $700 \mathrm{~nm}$ 、 光程 $10 \mathrm{~mm}$ 的石英比色血中测定了 POEGMA- $b-$ $\mathrm{P}(\mathrm{APUEMA}-\mathrm{co}-\mathrm{NBD})$ 水溶液在 NO 处理前后的透光率. 所有测量的聚合物浓度为 $1.0 \mathrm{~g} / \mathrm{L}$.

\section{5 体内实验}

用 0,100 和 $500 \mu \mathrm{mol} / \mathrm{L}$ 的二醇二氮烯鎓盐 (NONOate)处理 MCF-7 细胞 $2 \mathrm{~h}$ 后, 将基质移除, 用含 有 $1 \mu \mathrm{g} / \mathrm{mL}$ 的 $\mathrm{RNO}^{-}$的新鲜基质润洗细胞并孵育 $30 \mathrm{~min}$ 后移除并拍照. 对 MCF-5 细胞, 用 0 和 $500 \mu \mathrm{mol} / \mathrm{L}$ 的
NONOate 处理 $2 \mathrm{~h}$ 后, 将基质移除, 用含有 $1 \mu \mathrm{g} / \mathrm{mL}$ 的 $\mathrm{RNO}^{-}$的新鲜基质润洗细胞并孵育 $30 \mathrm{~min}$ 后移除并拍 照. 每批次处理均拍照至少 10 张并用 Image $\mathrm{J}$ 软件进行 苂光强度表征.

\section{References}

[1] Culotta, E.; Koshland, D. E. Science 1992, 258, 1862.

[2] Szabo, C. Nat. Rev. Drug Discovery 2007, 6, 917.

[3] Chen, X. Q.; Tian, X. Z.; Shin, I.; Yoon, J. Chem. Soc. Rev. 2011, 40 4783.

[4] Calabrese, V.; Mancuso, C.; Calvani, M.; Rizzarelli, E.; Butterfield, D. A.; Stella, A. M. Nat. Rev. Neurosci. 2007, 8, 766.

[5] Castegna, A.; Thongboonkerd, V.; Klein, J. B.; Lynn, B.; Markesbery, W. R.; Butterfield, D. A. J. Neurochem. 2003, 85, 1394.

[6] Carpenter, A. W.; Schoenfisch, M. H. Chem. Soc. Rev. 2012, 41, 3742 .

[7] Roseand, M. J.; Mascharak, P. K. Curr. Opin. Chem. Biol. 2008, 12, 238.

[8] Klahr, S. Nephrol. Dial. Transpl. 2001, 16(Suppl 1), 60.

[9] Cary, S. P. L.; Winger, J. A.; Derbyshire, E. R.; Marletta, M. A. Trends Biochem. Sci. 2006, 31, 231.

[10] Handbook of Experimental Pharmacology, Vol. 143, Ed.: Mayer, B., Springer, Berlin, 2000.

[11] Coneski, P. N.; Schoenfisch, M. H. Chem. Soc. Rev. 2012, 41, 3753.

[12] Bogdan, C. Nat. Immunol. 2001, 2, 907.

[13] Bogdan, C. Trends Immunol. 2015, 36, 161.

[14] Sun, Y. Q.; Liu, J.; Zhang, H.; Huo, Y.; Lv, X.; Shi, Y.; Guo, W. J. Am. Chem. Soc. 2014, 136, 12520.

[15] Sandipan, B. Y. R.; Shrabani, B.; Manoranjan, B.; Amrita, P.; Mahitosh, M.; Singh, N. D. P. Chem. Commun. 2018, 54, 7940.

[16] Mao, Z. Q.; Feng, W. Q.; Li, Z.; Zeng, L. Y.; Lv, W. J.; Liu, Z. H. Chem. Sci. 2016, 7, 5230.

[17] Deasai, A. V.; Samanta, P.; Manna, B.; Ghosh, S. K. Chem. Commun. 2015, 51, 6111.

[18] Lim, A. H.; Lippard, S. J. Acc. Chem. Res. 2007, 40, 41.

[19] Lim, M. H.; Xu, D.; Lippard, S. J. Nat. Chem. Biol. 2006, 2, 375

[20] Hu, X.; Wang, J.; Zhu, X.; Dong, D.; Zhang, X.; Wu, S.; Duan, C. Chem. Commun. 2011, 47, 11507.

[21] Chen, Y.; Guo, W.; Ye, Z.; Wang, G.; Yuan, J. Chem. Commun. 2011, 47, 6266.

[22] Luzio, L. P.; Pryor, P. R.; Bright, N. A. Nat. Rev. Mol. Cell. Biol. 2007, 8, 622 .

[23] Park, J. H.; Pramanick, S.; Kim, J.; Lee, J.; Kim, W. J. Chem. Commun. 2017, 53, 11229.

[24] Hu, J. M.; Whittaker, M. R.; Quinn, J. F.; Davis, T. P. Macromolecules 2016, 49, 2741.

[25] Zheng, D. B.; Gao, Z. F.; Xu, T. Y.; Liang, C. H.; Shi, Y.; Wang, L.; Yang, Z. M. Nanoscale 2018, 10, 21459.

[26] Nishikawa, Y.; Miki, T.; Awa, M.; Kuwata, K.; Tamura, T.; Hamachi, I. ACS Chem. Biol. 2019, 14, 397.

(Cheng, B.) 